\title{
The Thin Red Line between Pathological and Physiological Inflammatory Background in the Gastric Mucosa
}

\author{
Matteo Fassan ${ }^{\mathrm{a}, \mathrm{b}}$

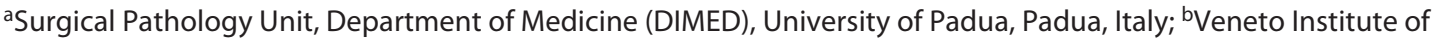 \\ Oncology, IOV-IRCCS, Padua, Italy
}

The immunological complexity of gastric mucosa was recognized and studied only after the discovery of Helicobacter pillory (Hp) and its associated carcinogenetic cascade $[1,2]$. In this setting, several factors have impaired the definition of the physiological inflammatory infiltrate characterizing gastric mucosa.

Most of the samples analyzed in our surgical pathology departments are derived from dyspeptic patients, in which an organic (mainly longstanding inflammatory) disease determined the clinical presentation, and, thus, they cannot be considered as "standard" controls. Of note, once the pathogenetic insult (e.g., Hp eradication) has been removed, chronic inflammatory cells have been shown to slowly decrease and it takes several months/ years to revert gastric mucosa status back into to a physiological state [3].

Moreover, even in the absence of any inflammatory injury, the gastric immunological milieu is the result of a complex overlap of environmental, demographic, dietary, and genetic factors, which lead to an intrinsic inflammatory infiltration of the lamina propria [1]. Several variables can influence the number and composition of inflammatory cells present within the gastric mucosa. Therefore, it is difficult to define the real border between

karger@karger.com

(c) 2021 S. Karger AG, Basel

www.karger.com/ddi

Karger! a physiological situation and a mild inflammatory condition.

The central matter is whether the use of the term "normal" is appropriate when referring to the presence of inflammatory cells in the gastric mucosa. As suggested by the updated Sydney system [4], we should refer to an "expected" rather than a "normal" level of chronic inflammatory cell infiltration. In control subjects, the gastric mucosa contains only individual scattered chronic inflammatory cells (i.e., lymphocytes, plasma cells, and macrophages) in the lamina propria. Occasional intraepithelial lymphocytes may also be observed in the surface epithelium. According to the updated Sydney system, the detection of 2-5 lymphocytes, plasma cells, and macrophages per high-power microscope field or 2-3 of them between foveolae can be regarded as physiological [4]. However, giving an appropriate definition of mild gastritis can be challenging and is subject to significant interobserver agreement variation $[5,6]$.

Sumi et al. [7] have systematically investigated the exact number of inflammatory cells in stomach biopsies obtained from a relatively large $(n=325)$ cohort of Japanese subjects with endoscopically non-pathological mucosa and without $\mathrm{Hp}$ infection. 
Of note, they found a higher number of inflammatory cells in the antral compartment in comparison to fundic gland mucosa. This finding further supports the importance of a separate histopathological evaluation of the two gastric compartments, which are indeed characterized by different histological, pathological, and functional aspects. On the other hand, we must consider the possible influence of the inflammatory insult derived by duodenogastric reflux, which can occur as a sub-clinical disease.

Surprisingly, an interesting finding was the lack of ageor sex-based differences in the number of inflammatory cells. In particular, age should ideally reflect the exposure of the gastric mucosa to dietary habits, medications, cigarette smoking, and other environmental factors that can influence the intensity of immune cell infiltration. However, this finding may indicate that only a direct damage on the gastric mucosa (such as Hp infection) may significantly alter the steady immunological state.

The establishment of reliable endoscopic and clinical criteria for the selection of control subjects and the introduction of comprehensive molecular profiling technologies should be applied in the definition of the baseline immunological background of gastric mucosa. This would help translational research in the field of secondary prevention strategies for gastric cancer and better understand the therapeutic scenario related to the novel immune modulatory therapies.

\section{Conflict of Interest Statement}

Matteo Fassan received research funding from Astellas Pharma, Macrophage Pharma, and QED Therapeutics and had roles as consultant or advisor for Astellas Pharma, GSK-Tesaro, MSD, Roche, and Diaceutics. The author has no conflicts of interest to declare regarding the present editorial.

\section{Funding Sources}

Matteo Fassan is supported by a grant from the Italian Health Ministry/Veneto region research program NET-2016-02363853 and AIRC 5 per mille 2019 (ID. 22759 program). The funding agencies had no role in the writing of the editorial.

\section{Author Contributions}

M.F. wrote the paper.

\section{References}

1 Hunt RH, Camilleri M, Crowe SE, El-Omar EM, Fox JG, Kuipers EJ, et al. The stomach in health and disease. Gut. 2015 Oct;64(10): 1650-68.

2 Pennelli G, Grillo F, Galuppini F, Ingravallo G, Pilozzi E, Rugge M, et al. Gastritis: update on etiological features and histological practical approach. Pathologica. 2020 Sep;112(3): 153-65.

3 Genta RM, Lew GM, Graham DY. Changes in the gastric mucosa following eradication of helicobacter pylori. Mod Pathol. 1993 May; 6(3):281-9.
4 Dixon MF, Genta RM, Yardley JH, Correa P. Classification and grading of gastritis. The updated Sydney system. International workshop on the histopathology of gastritis, Houston. Am J Surg Pathol. 1996 Oct;20(10):116181.

5 Andrew A, Wyatt JI, Dixon MF. Observer variation in the assessment of chronic gastritis according to the Sydney system. Histopathology. 1994 Oct;25(4):317-22.
6 Aydin O, Egilmez R, Karabacak T, Kanik A Interobserver variation in histopathological assessment of helicobacter pylori gastritis. World J Gastroenterol. 2003 Oct;9(10):22325.

7 Sumi N, Haruma K, Kamada T, Suehiro M, Manabe N, Akiyama T, et al. Inflammatory cell numbers in the stomach of Japanese subjects with endoscopically normal mucosa without helicobacter pylori infection. Dig Dis. 2021;39(6):598-605. 